
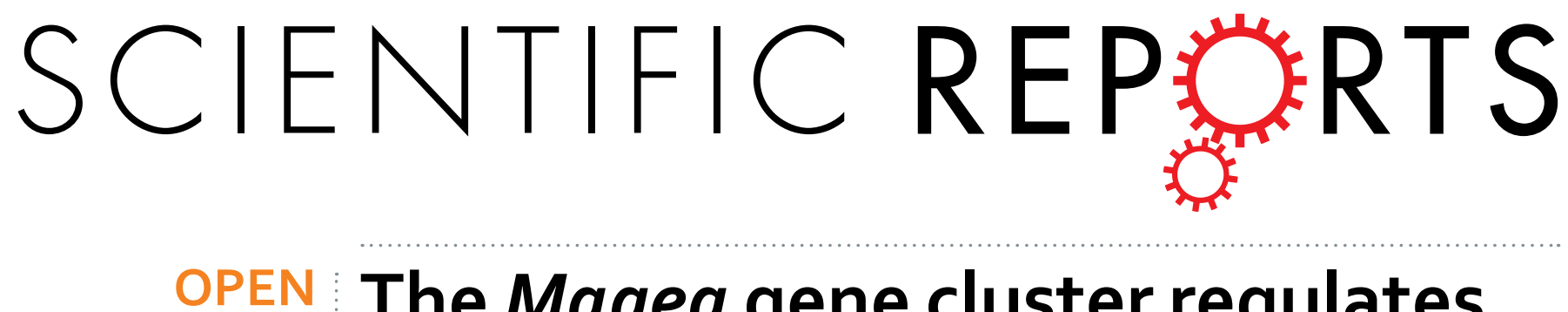

\title{
The Magea gene cluster regulates male germ cell apoptosis without affecting the fertility in mice
}

Received: 21 January 2016

Accepted: 06 May 2016

Published: 26 May 2016
Siyuan Hou*, Li Xian*, Peiliang Shi, Chaojun Li, Zhaoyu Lin \& Xiang Gao

While apoptosis is essential for male germ cell development, improper activation of apoptosis in the testis can affect spermatogenesis and cause reproduction defects. Members of the MAGE-A (melanoma antigen family A) gene family are frequently clustered in mammalian genomes and are exclusively expressed in the testes of normal animals but abnormally activated in a wide variety of cancers. We investigated the potential roles of these genes in spermatogenesis by generating a mouse model with a 210-kb genomic deletion encompassing six members of the Magea gene cluster (Magea1, Magea2, Magea3, Magea5, Magea6 and Magea8). Male mice carrying the deletion displayed smaller testes from 2 months old with a marked increase in apoptotic germ cells in the first wave of spermatogenesis. Furthermore, we found that Magea genes prevented stress-induced spermatogenic apoptosis after $\mathrm{N}$-ethyl-N-nitrosourea (ENU) treatment during the adult stage. Mechanistically, deletion of the Magea gene cluster resulted in a dramatic increase in apoptotic germ cells, predominantly spermatocytes, with activation of $\mathrm{p} 53$ and induction of $\mathrm{Bax}$ in the testes. These observations demonstrate that the Magea genes are crucial in maintaining normal testicular size and protecting germ cells from excessive apoptosis under genotoxic stress.

Apoptosis is a highly regulated and conserved mechanism that plays a critical role in the development and homeostasis of multicellular organisms $s^{1,2}$. A large number of studies have demonstrated the major importance of apoptosis during male germ cell development ${ }^{3}$. Spermatogenesis is a complex process of male germ cell proliferation and differentiation from diploid spermatogonia to haploid mature functional sperm, which can fertilize the ovum and transmit genetic information to the next generation. However, only $25 \%$ of all spermatogonial progeny are estimated to become mature spermatozoa, thus leaving up to $75 \%$ to be eliminated by germ cell apoptosis ${ }^{4-6}$. Both spontaneous and stress-induced apoptosis play essential roles in normal germ cell development, and these processes are required for maintaining testicular homeostasis and a critical cell population ratio between Sertoli cells and maturing germ cells as well as for maintaining the integrity of the germ-line genome by clearing the mutational load during the first meiotic division ${ }^{5,6}$. Dysregulation of germ cell apoptosis, in turn, may cause suboptimal male reproductive function and even male infertility ${ }^{4,6}$. Moreover, increased germ cell apoptosis has been shown to be induced by various environmental stresses, such as toxicant exposure, temperature change, hormonal depletion, radiation and oxidative stress ${ }^{7-10}$. In this regard, exposure to environmental toxicants has been suggested to cause male reproductive defects. Additionally, although the survival of cancer patients has increased with the use of radiation and chemotherapeutic drugs, the treatments may cause germ cell apoptosis and male fertility problems ${ }^{11}$. Consequently, there is a growing need to investigate the mechanisms of spermatogenic apoptosis regulation and to identify ways to prevent excessive male germ cell apoptosis.

The melanoma antigen (MAGE) genes are conserved in all eukaryotes and share a common MAGE homology domain (MHD) domain with high sequence similarity. Almost two-thirds of all MAGEs are regarded as cancer/ testis antigens $(\mathrm{CTA})^{12}$. CTAs are a group of proteins that are typically expressed in germ cells and trophoblast lineages, but they are abnormally expressed in various types of cancer ${ }^{13}$. The first cancer testis antigen, MAGEA1, was discovered because it could be recognized by cytotoxic $\mathrm{T}$ cells in patients with malignant melanoma ${ }^{14}$. The MAGE-A family consists of 12 members that are classified as type I MAGE proteins and are located on chromosome Xq28 in humans ${ }^{12}$. The MAGE-A proteins are abnormally activated in a wide variety of tumours, including

State Key Laboratory of Pharmaceutical Biotechnology and MOE Key Laboratory of Model Animal for Disease Study, Model Animal Research Center, Nanjing Biomedical Research Institute, Nanjing University, China. *These authors contributed equally to this work. Correspondence and requests for materials should be addressed to Z.L. (email: linzy@nju.edu.cn) orX.G. (email: gaoxiang@nju.edu.cn) 
melanoma, pancreatic cancer, breast cancer, non-small cell lung cancer, and ovarian carcinoma ${ }^{13-15}$, and they are especially associated with invasion, metastasis, poor patient survival, and an aggressive clinical course ${ }^{16-18}$. Recent evidence has suggested that MAGE-A proteins might be important and could serve as transcriptional regulators and key components of the ubiquitin ligase complex in cancer cells $\mathrm{s}^{19-21}$. Furthermore, several in vitro cell studies have demonstrated that MAGE-A antigens possess tumourigenic features that promote malignant transformation, cell proliferation and tumour survival and formation by inhibiting p53 transactivation and suppressing p53-dependent apoptosis ${ }^{22-24}$.

Eight members of the MAGE-A gene cluster (Magea1-Magea8) are also expressed throughout the spermatogenic series (from spermatogonia to primary pachytene spermatocytes) during the meiotic division and proliferation stages ${ }^{25}$. MAGE-A proteins (MAGEA1, A3, A4, A6 and A12) are present in the nuclei and cytoplasm of spermatogonia and spermatocytes in adult testes ${ }^{13}$ and Magea mRNAs (Magea3 and Magea5) are detected through the first wave of spermatogenesis in juvenile male mice ${ }^{26}$. MAGE-A proteins (MAGEA1, A2, A3, A4, A6, $\mathrm{A} 10$ and A12) are also expressed in migrating primordial germ cells in 5-week-old human embryo ${ }^{27}$. This expression pattern of the MAGE-A genes indicated that they could be involved in the initialization and progression of spermatogenesis. Researchers have focused heavily on developing tumour vaccines for cancer immunotherapy, and a number of MAGE-A peptides and recombinant proteins have been used in clinical trials ${ }^{17,18}$ because the expression of $M A G E-A$ genes is normally restricted to the testes and these proteins have served as tumour-specific antigens in a wide range of cancers ${ }^{28}$. However, the in vivo function of the Magea gene family remains largely unknown and understudied.

Here, we report a new mouse model carrying targeted deletions of six members of the Magea gene cluster. We showed that Magea genes protected germ cells from excessive apoptosis under conditions of both physiological and N-ethyl-N-nitrosourea (ENU)-induced genotoxic stress. This study is the first to highlight the in vivo function of Magea genes during male germ cell development.

\section{Results}

Generation of a mouse model with targeted deletions of Magea genes. The Magea gene family in mice consists of 10 members (Magea1 to a10). They are located in two subsets on chromosome XqF3 (Magea1, $a 2, a 3, a 5, a 6$ and $a 8$ ) and XqA7.3 (Magea4, a7, a9 and a10). Based on previous studies, Magea7 and $a 9$ are both pseudogenes that are not expressed, and the expression of Magea4 is weak in mouse testes. In addition, phylogenetic conservation analysis suggested that the major cluster on chromosome $\mathrm{XqF} 3$ is evolutionarily distinct from the minor cluster and confers functionality to the Magea gene family (Supplementary Fig. S1A). Therefore, we generated a knockout mouse model by targeted removal of the Magea cluster on chromosome XqF3 with the aim of identifying the roles of Magea genes in male germ cell development.

To delete the 210-kb genomic region on chromosome XqF3, two loxp sites flanking this region were introduced upstream of Magea1 and downstream of Magea6 (Fig. 1A and supplementary Fig. S1B-G). The Magea-null mice were obtained by breeding Magea $^{\text {flox/+ }}$ females with EIIa-cre male mice to generate a constitutive knockout allele of the Magea cluster (Magea ${ }^{-/ Y}$ ) in the one-cell zygote stage of embryonic development ${ }^{29}$. The presence of this large deletion was confirmed by PCR genotyping (Supplementary Fig. S1H). RT-PCR analysis showed that the expression of the six members of the Magea gene cluster was undetectable in hemizygous mice $\left(\right.$ Magea $\left.^{-/ Y}\right)$ (Supplementary Fig. S1I). Magea ${ }^{-/ Y}$ mice appeared normal, and their total body mass and different organ masses (the brain, heart, liver, spleen and kidneys) were similar to those of their control littermates, indicating that the deletion of the Magea gene cluster did not affect overall postnatal growth (Supplementary Fig. S2A,B). In addition, genotyping of offspring from the cross between $\mathrm{Magea}^{+/ \mathrm{Y}}$ and $\mathrm{Magea}^{-1+}$ revealed a normal Mendelian ratio in the obtained mice, suggesting that deletion of the six members of the Magea gene cluster did not affect the survival of the embryos (Supplementary Table S1).

Deletion of the Magea gene cluster results in smaller testes and increased apoptosis in the first wave of spermatogenesis. We investigated the function of the Magea gene cluster during testicular development. We measured the size of the testes of $\mathrm{Magea}^{-/ Y}$ mice from the prepubertal period to 25 months of age. A significantly decreased testis mass was observed as early as 2 months of age, but not at postnatal days 12 (P12), 20 (P20), or 30 (P30) (Fig. 1B,C). The testis weights of the Magea $^{-/ Y}$ mice at 3, 5, 11 and 25 months of age were also significantly reduced compared with those of their wild-type littermates (Fig. 1C). The average testicular mass to body mass ratio $(\mathrm{mg} / \mathrm{g})$ of the $\mathrm{Magea}^{-/ \mathrm{Y}}$ mice was $16.6 \%\left(\mathrm{n}=10, \mathrm{p}=9.46 \times 10^{-3}\right)$ lower than that of the wild-type mice at the same age. To investigate whether spermatogenesis was impaired in the Magea $^{-/ Y}$ mice, we analyzed testicular histology using haematoxylin and eosin (H\&E) staining (Fig. 2A). Pathological analysis of the testes of 3-month-old Magea $^{-/ Y}$ mice revealed a weak but significant reduction in the area, diameter and perimeter of the seminiferous tubules (Fig. 2B-D). Moreover, a significant decrease $(n=8, p=0.0006)$ in the testicular sperm reserve of the Magea-null mice was observed compared with wild-type controls at 2 months of age (Supplementary Fig. S3A). The reduction in testis weight, seminiferous tubule diameter and sperm reserve of Magea $^{-/ Y}$ mice suggested that specific defects occurred during the first wave of spermatogenesis.

The first wave of spermatogenesis, which begins at P5 in mice, is accompanied by a wave of apoptosis that peaks 10-30 days after birth ${ }^{5}$. Germ cells enter meiotic prophase at approximately P10; subsequently, spermatocytes start to appear and enter the zygotene/pachytene transition at $\mathrm{P} 12^{30}$. At this time point, spermatocytes could be eliminated via apoptosis at the pachytene checkpoint. This first wave of germ cell apoptosis at approximately P12, which occurs mostly in spermatocytes, is important for functional spermatogenesis in adulthood ${ }^{4}$. During normal spermatogenesis in mammals, it is estimated that up to $75 \%$ of spermatogonial descendants have been eliminated by germ cell apoptosis ${ }^{4-6}$. Hence, we hypothesized that smaller testes and reduced seminiferous tubule area in Magea-null mice at 2 months and later might result from an earlier increase in apoptosis at P12. Consistent with this hypothesis, a marked increase in TUNEL-positive (terminal deoxynucleotidyl transferase dUTP nick 
A

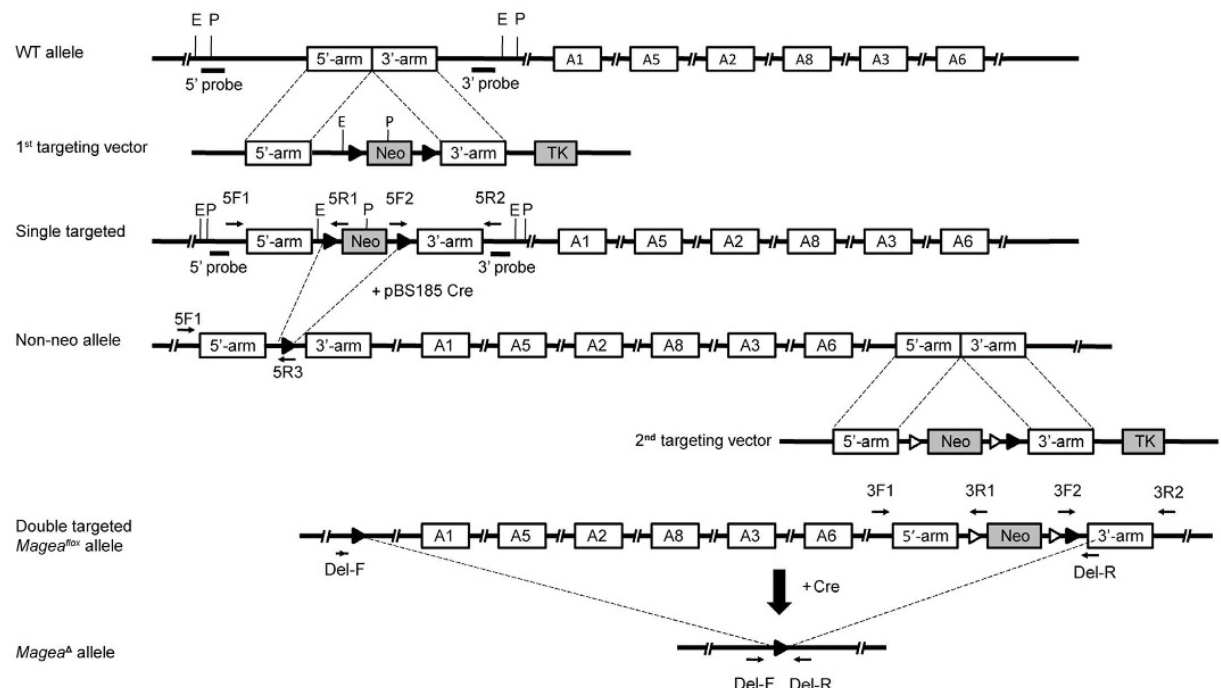

B

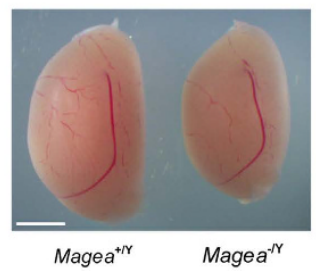

C

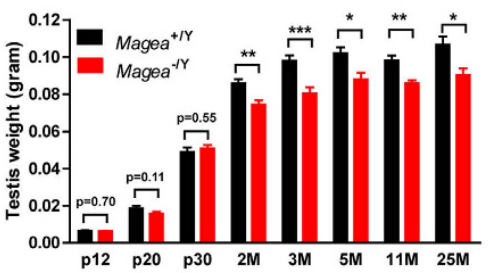

Figure 1. Deletion of the Magea gene cluster resulted in smaller testes. (A) Schematic representation of the wild-type Magea allele, the upstream $\left(1^{\text {st }}\right)$ and downstream $\left(2^{\text {nd }}\right)$ targeting vector, the single targeted allele, double targeted allele and the Magea-null allele. Six Magea genes are represented by open boxes in which each gene name is indicated (A1, Magea1; A2, Magea2; A3, Magea3; A5, Magea5; A6, Magea6; A8, Magea8. Probes (solid black line) and primers (arrow mark) for detecting homologous recombination via Southern blot and PCR analysis are denoted. EcoRI (E) and PstI (P) restriction enzyme sites are indicated. loxp sites (blank triangles), frt sites (open triangles) and the $5^{\prime}$ and $3^{\prime}$ recombination arms of targeting vectors are shown. (B) The testes of $\mathrm{Magea}^{-/ \mathrm{Y}}$ mice were smaller than those of wild-type mice at the age of 3 months. Scale bar, $2 \mathrm{~mm}$. (C) Testis weights of Magea $^{-/ \mathrm{Y}}$ and wild-type littermates were determined at the postnatal ages of $12 \mathrm{~d}$ (P12), $20 \mathrm{~d}$ (P20), $30 \mathrm{~d}$ (P30), 2 months (2 M), 3 months (3 M), 5 months (5 M), 11 months (11 $\mathrm{M}$ ) and 25 months $(25 \mathrm{M}) . \mathrm{N}=3,4,5,8,10,6,4$ and 5 for P12, P20, P30, $2 \mathrm{M}, 3 \mathrm{M}, 5 \mathrm{M}, 11 \mathrm{M}$ and $25 \mathrm{M}$, respectively. ${ }^{* *} \mathrm{p}<0.001 ;{ }^{* *} \mathrm{p}<0.01 ;{ }^{\star} \mathrm{p}<0.05$.

end labeling assay) cells was observed in the Magea $^{-/ Y}$ testes (Fig. 2F,G) at P12, although no differences were observed in the histological analysis (Supplementary Fig. S4A). Because most of the apoptotic cells were located in the middle of the seminiferous tubules, they were presumed to be primary spermatocytes (Fig. 2F). Co-staining of markers for spermatocytes (SCP3) and apoptosis (activated caspase-3) confirmed increased apoptosis in spermatocytes (Fig. 2H,I). The presence of spermatogonia and spermatocytes was demonstrated by immunofluorescence double staining of Plzf and SCP3, indicating normal differentiation from spermatogonia to spermatocytes (Supplementary Fig. S4B). Ki67 immunohistochemistry showed that there was no significant difference in the average number of Ki67-positive cells between the Magea $^{-/ Y}$ mice and wild-type controls, suggesting that spermatogonia in seminiferous tubules exhibit a normal proliferation rate (Supplementary Fig. S4C,D). Collectively, these findings suggest that the Magea genes play an indirect role in maintaining normal testicular size and prevent spermatocytes from undergoing apoptosis during the first wave of spermatogenesis (P12) in male mice.

Magea genes prevent excessive apoptosis in the testes under genotoxic stress induced by ENU. We analyzed the impact of the observed defects in $\mathrm{Magea}^{-/ \mathrm{Y}}$ testes on sperm production and testicular apoptosis, and found that the sperm count (Supplementary Fig. S5B,C, using two different methods and a slightly decrease observed using the latter method), sperm motility (Supplementary Fig. S5D-I), reproductive ability (Supplementary Table S2) and testicular apoptosis (Supplementary Fig. S6) of these mice were similar to those of wild-type controls. Although normal fertility and testicular apoptosis were observed in Magea $^{-/ \mathrm{Y}}$ mice, dramatically alterations in spermatogenesis may be detected under stress conditions, including environmental toxicants or chemicals. We therefore sought to determine the role of the Magea gene cluster on germ cells apoptosis in the response to genotoxic stress induced by the DNA-damaging agent $\mathrm{N}$-ethyl-N-nitrosourea (ENU). ENU belongs to the family of $\mathrm{N}$-nitroso compounds (NOCs), which are regarded as environmental toxicants. Humans are exposed to NOCs in the environment through certain foods, tobacco smoking and industrial products, and NOCs are widely considered as biological hazards on male reproduction ${ }^{31}$. Exposure to ENU in male mice produces severe disruption of adult spermatogenesis and leads to a significant reduction in testis weight and sperm 
A

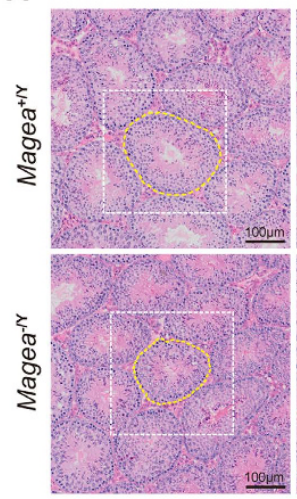

H
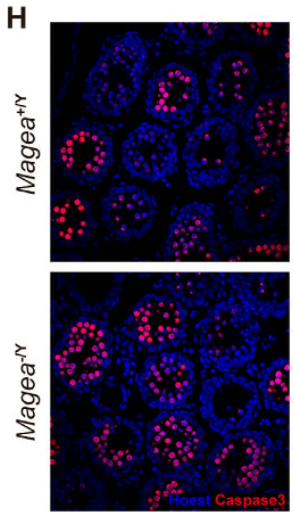

B
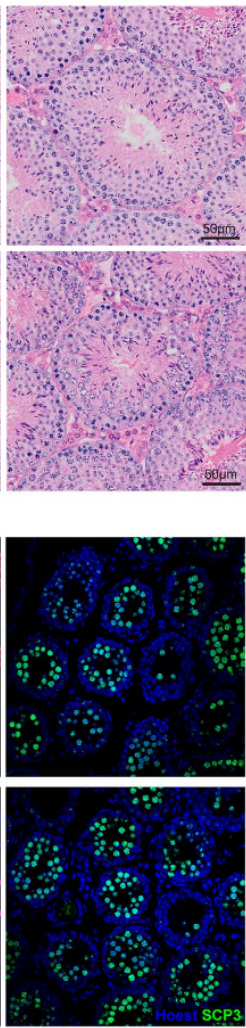

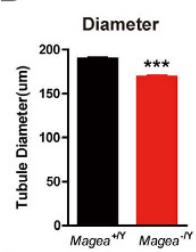

D Perimeter $\mathrm{E}$
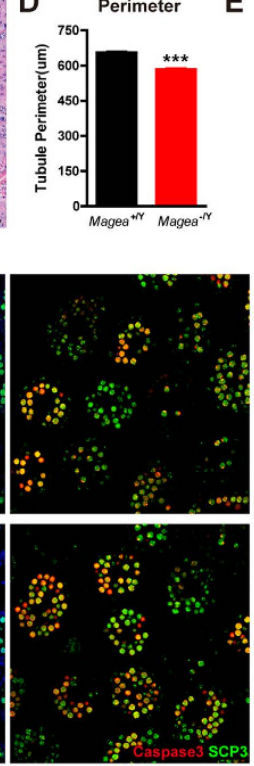

C

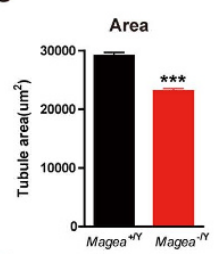

E
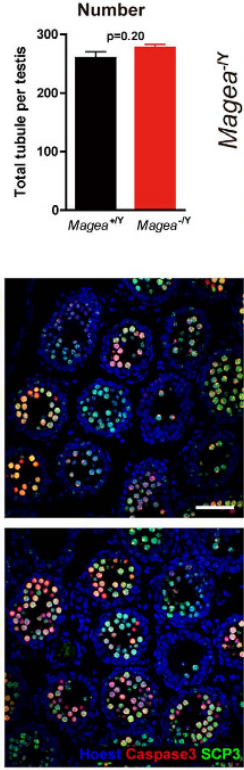

$\mathbf{F}$

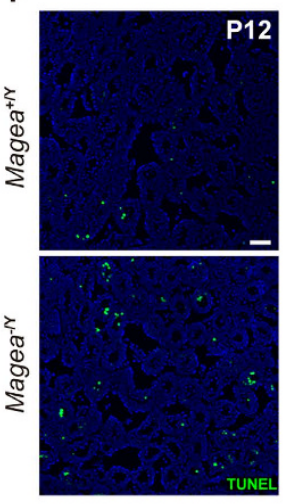

G

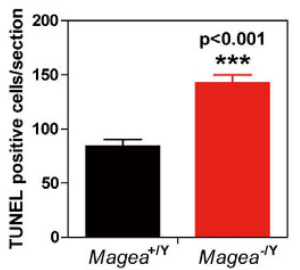

I

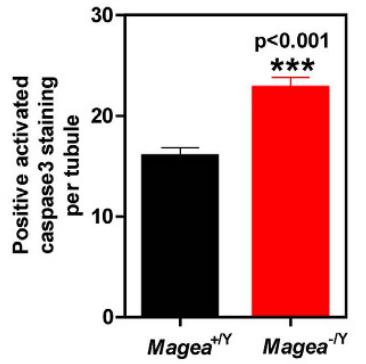

Figure 2. Deletion of the Magea gene cluster led to an increase in apoptosis in the first wave of spermatogenesis in Magea $^{-/ Y}$ testes. (A) Haematoxylin-eosin sections of the testes of 3-month-old mice. Compared with wild-type testes, the area and diameter of the seminiferous tubules of the testes of $\mathrm{Magea}^{-/ \mathrm{Y}}$ mice were significantly smaller. (B-E) Quantification and characterization of seminiferous tubules including the diameter, area, perimeter and total number of seminiferous tubules $\left(\mathrm{n}=188\right.$ for wild-type and $\mathrm{n}=124$ for $\left.\mathrm{Magea}^{-/ \mathrm{Y}}\right)$. (F) An increase in the number of apoptotic cells was observed in the testes of $\mathrm{Magea}^{-/ \mathrm{Y}}$ mice at the postnatal age of 12 days (P12) by TUNEL staining. Hoechst 33342 staining revealed the nuclei morphology. Scale bar, $50 \mu \mathrm{m}$. (G) Quantification of apoptotic cells in wild-type and $\mathrm{Magea}^{-/ \mathrm{Y}}$ testes at the postnatal age of 12 days (P12). Apoptotic cells were counted in 9 sections for each genotype. (H) Immunofluorescence of activated caspase-3 and SCP3 in testicular sections of Magea $^{-/ Y}$ mice at the postnatal age of 12 (P12) days. Scale bar, $50 \mu \mathrm{m}$. (I) Quantification of cells with positive activated caspase-3 staining in each seminiferous tubule $\left(\mathrm{n}=180\right.$ for wild-type and $\mathrm{n}=222$ for $\left.\mathrm{Magea}^{-/ \mathrm{Y}}, \mathrm{p}<0.001\right) .{ }^{* *} \mathrm{p}<0.001$.

count due to germ cell apoptosis and growth arrest ${ }^{32}$. First, mice were given three daily ENU $(20 \mathrm{mg} / \mathrm{kg})$ intraperitoneal (i.p.) injections (accumulated dose, $60 \mathrm{mg} / \mathrm{kg}$ ENU). Testicular histology was evaluated in the Magea $^{-/ Y}$ mice and wild-type controls 1 week after the first ENU injection. H\&E staining showed that the seminiferous tubules in the testes of the $\mathrm{Magea}^{-/ \mathrm{Y}}$ mice contained more apoptotic cells, which have a condensed, dark red cytoplasm and dark purple pyknotic nuclei (Fig. 3A). TUNEL staining confirmed significantly increased apoptosis in the testes of the Magea ${ }^{-/ Y}$ mice compared with their wild-type littermates (Fig. 3B,C). Immunofluorescence double staining of SCP3 and activated caspase- 3 demonstrated that these apoptotic cells were mainly spermatocytes, and the quantification of cells with positive caspase- 3 staining, which revealed increased apoptosis, further supported the results of TUNEL staining (Fig. 3D,E). Furthermore, the results of flow cytometry analysis of Annexin V-FITC and propidium iodide (PI) double staining revealed that the proportion of apoptotic cells was significantly increased in Magea $^{-/ Y}$ mice (Fig. 3F,G). Previous studies have demonstrated that ENU can induce apoptosis as early as 3 hours after ENU treatment in the fetal central nervous system and in the trophoblastic cells of the placenta ${ }^{33,34}$. We determined whether germ cells responded in an acute manner and whether Magea-null mice responded more intensely in the early stages after ENU treatment. Flow cytometry analysis of a 7-day time course of apoptosis showed that the percentage of germ cells undergoing apoptosis peaked on day 1, suggesting an acute apoptotic response after ENU-induced genotoxic stress (Fig. 4A,B). Then the apoptotic change in the early stages after ENU administration was examined in the testes of the Magea knockout mice. The result of flow cytometry analysis revealed that the proportions of apoptotic cells (Annexin V-FITC positive) and dead cells (Annexin V-FITC negative and propidium iodide positive) were significantly increased in Magea-null testes compared to $\mathrm{Magea}^{+/ \mathrm{Y}}$ testes 24 hours after ENU treatment, although no difference was observed 12 hours after ENU treatment between Magea-null testes and wild-type controls (Fig. 4C,D). Together, these results suggest that Magea genes are functionally important for protecting spermatocytes from excessive apoptosis in an acute manner after ENU-induced genotoxic stress at 3 months of age. 
A

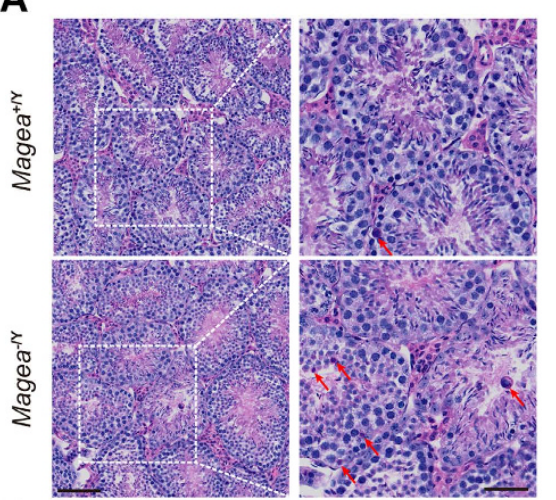

D
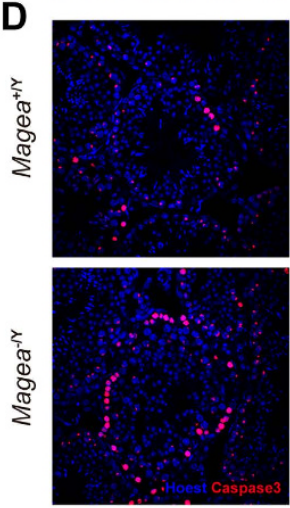

$\mathbf{F}$
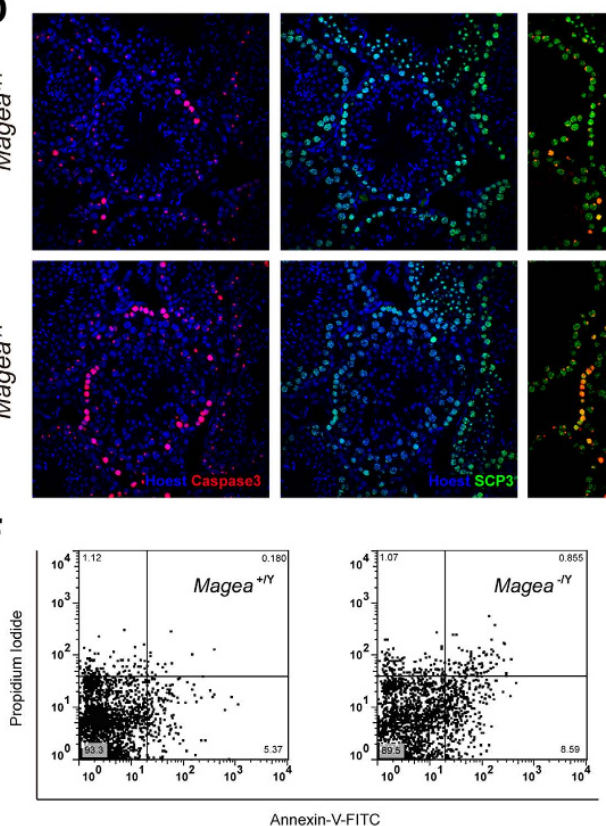

B
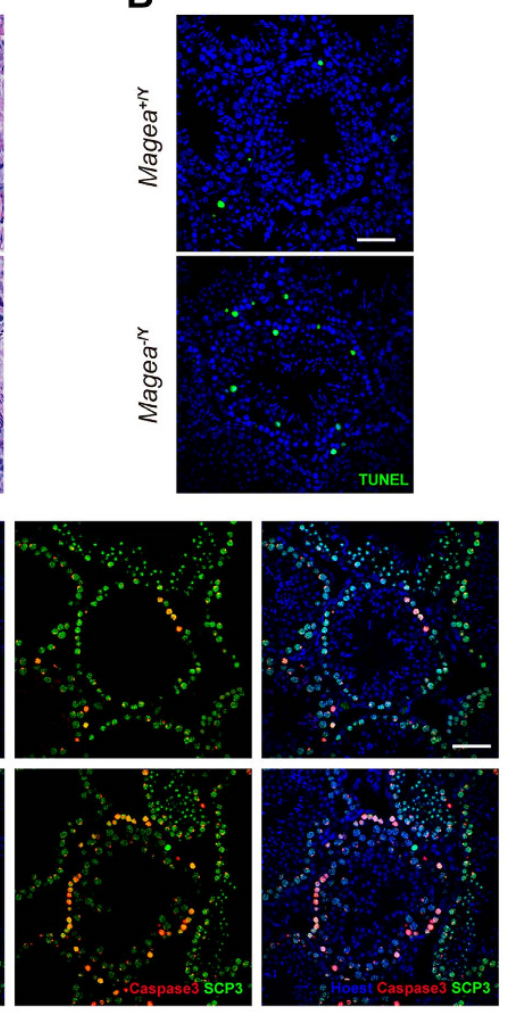

G

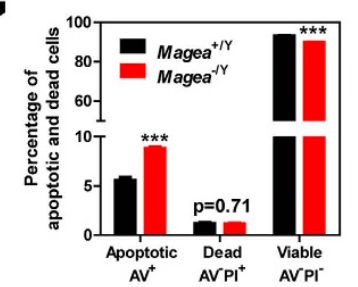

C

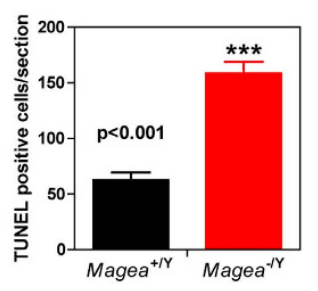

E

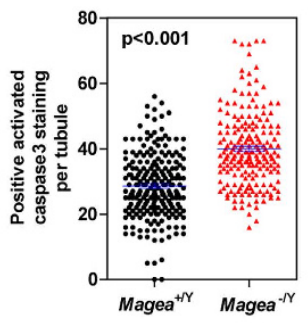

Figure 3. Magea genes protected germ cells from apoptosis after genotoxic stress induced by ENU.

(A) Testicular sections of 3-month-old wild-type and $\mathrm{Magea}^{-/ Y}$ mice 1 week after ENU treatment (late response assay). Scale bar, $100 \mu \mathrm{m}$. Further enlarged images of the areas in dashed boxes are shown in the right panels. Red arrows indicate apoptotic cells. Scale bar, $50 \mu \mathrm{m}$. (B,C) Increased numbers of apoptotic cells were observed in $\mathrm{Magea}^{-/ \mathrm{Y}}$ mice by TUNEL staining. Apoptotic cells were counted in 9 whole sections for each genotype. Scale bar, $50 \mu \mathrm{m}$. (D) Immunofluorescence of activated caspase- 3 and SCP3 in testicular sections of Magea ${ }^{-1 Y}$ mice. Scale bar, $50 \mu \mathrm{m}$. (E) Quantification of cells with positive activated caspase-3 staining in each seminiferous tubule ( $\mathrm{n}=223$ for wild-type and $\mathrm{n}=202$ for $\left.\mathrm{Magea}^{-/ \mathrm{Y}}, \mathrm{p}<0.001\right)$. (F,G) Representative flow cytometry dot plot of Annexin V-FITC and PI staining for the detection of testicular apoptosis $(\mathrm{n}=6)$. Magea $^{-/ Y}$ mice showed significantly increased apoptosis after exposure to ENU for 1 week. ${ }^{* * *} \mathrm{p}<0.001$.

Magea proteins inhibit $\mathrm{p} 53$ protein expression after genotoxic stress. The protein $\mathrm{p} 53$ is essential for meiotic checkpoint control, and acts as a guardian of the germ-line genome under physiological or stress conditions by clearing abnormal germ cells with mutated DNA by apoptosis ${ }^{35-37}$. Recent studies showed that Magea can promote $\mathrm{p} 53$ degradation and inhibit $\mathrm{p} 53$ function in tumour cells, providing cancer cells with a survival advantage ${ }^{20-24}$. Therefore, we investigated whether Magea proteins inhibit the expression of $\mathrm{p} 53$ in testes under genotoxic stress. In response to genotoxic stresses, such as ENU-induced DNA damage, the p53 signalling pathway can be activated, triggering apoptosis and growth arrest ${ }^{33,34,38}$. Consistent with previous studies, we found that the p53 signalling pathway was activated in the testes after ENU treatment. We showed that the mRNA levels of p53 and cyclin G1 were elevated 6 hours after ENU treatment (Supplementary Fig. S7A and Table S3). In addition, phosphorylated $\mathrm{p} 53$ (Ser15) and p21 protein levels were higher at 3 hours after ENU-induced DNA damage than at the zero time point after treatment with solvent (Fig. 5A,B). Subsequently, we tested whether loss of the Magea genes led to increased p53 protein levels and promoted its downstream signalling, resulting in a marked increase in apoptotic germ cells. The mRNA levels of Bax and Noxa in the testes of $\mathrm{Magea}^{-/ \mathrm{Y}}$ mice at the 3-hour point and Caspase-3 at the 6-hour point after ENU treatment were higher than those in the wild-type control mice (Supplementary Fig. S7B-D and Supplementary Table S3). Under physiological conditions, the protein level of p53 and Bax in the testes of the Magea-null mice was comparable to that of the wild-type controls (Fig. 5C). 
A

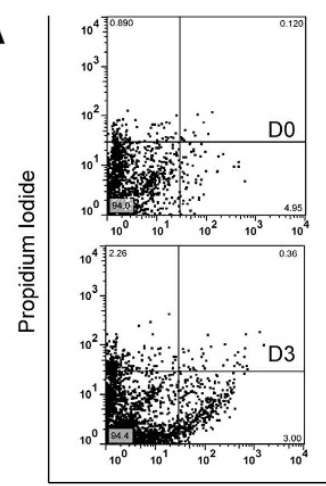

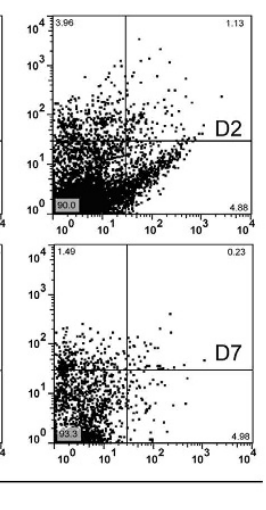

B

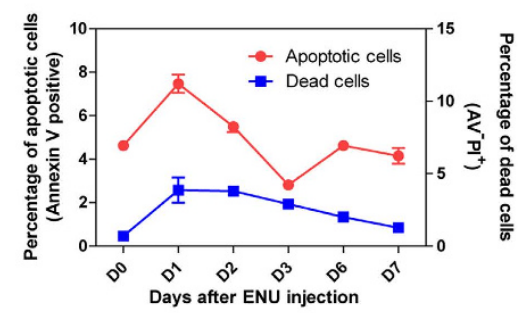

C
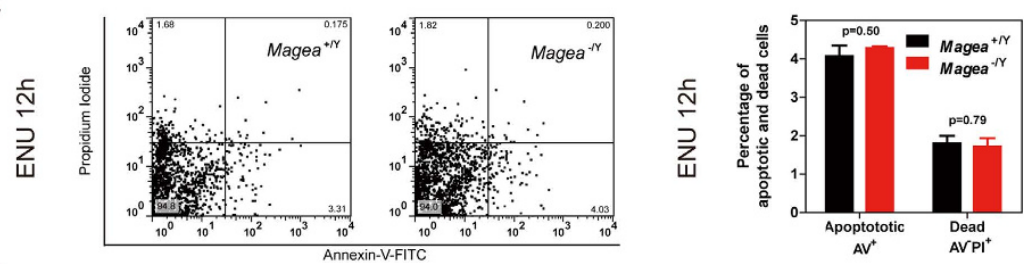

D
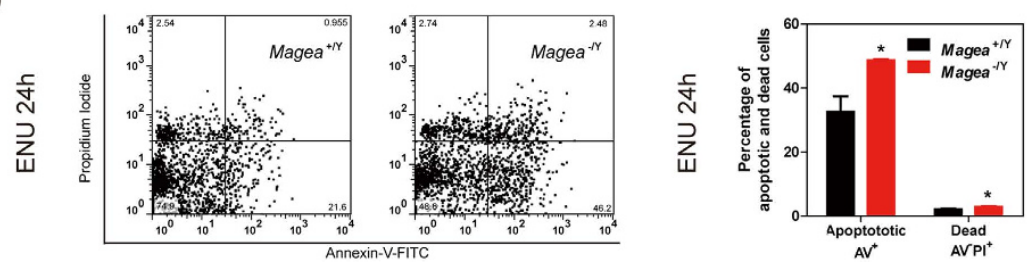

Figure 4. Magea proteins prevented an excessive apoptotic response in germ cells in an acute manner after ENU-induced genotoxic stress. (A) Time course flow cytometry analysis of testicular apoptosis in C57BL/6J mice after exposure to ENU. The results are shown at several time points (in days) as indicated. (B) Quantification of apoptosis after exposure to ENU for 1 week ( $\mathrm{n}=4$ for each time point). (C,D) Detection of testicular apoptosis by flow cytometry analysis at 12 hours and 24 hours after exposure to ENU $(60 \mathrm{mg} / \mathrm{kg})(12$ hours, $\mathrm{n}=4$ for wild type

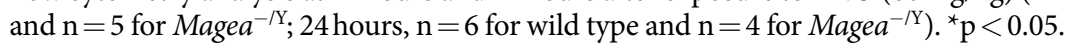

However, under genotoxic stress, the p53 protein level was much higher in the testes of the $\mathrm{Magea}^{-/ Y}$ mice 3 hours after ENU administration and was found to be more phosphorylated (Ser15) at 6 hours after ENU treatment (Fig. 5D,E and Supplementary Table S3). Bax, a direct downstream target of $\mathrm{p} 53$ that plays essential roles in germ cell apoptosis and testicular development ${ }^{4}$, showed a marked increase at 6 hours after ENU treatment in the Magea $^{-Y Y}$ mice (Fig. 5F,G and Supplementary Table S3). Taken together, these results suggest that Magea proteins inhibit the expression of $\mathrm{p} 53$ protein under ENU-induced genotoxic stress and prevent an excessive apoptotic response in male germ cells at 3 months of age.

\section{Discussion}

In our study, we successfully generated a novel mouse model with a 210-kb deletion encompassing six members of the Magea gene cluster (Magea1, a2, a3, a5, a6 and a8). Disruption of this gene cluster resulted in significant reductions in the size of the testes and the diameter of seminiferous tubules in $\mathrm{Magea}^{-/ Y}$ mice compared with wild-type mice. Furthermore, deletion of the Magea gene cluster led to an increase in the first wave of testicular apoptosis under normal conditions. We also observed increased apoptosis in spermatocytes with activation of p53 and induction of Bax in the testes of Magea knockout mice after ENU-induced genotoxic stress, suggesting that the Magea genes could inhibit the activation of p53 signalling to protect germ cells from excessive apoptosis.

During spermatogenesis, apoptosis play a pivotal role in maintaining homeostasis of different cell types and eliminating germ cells that are defective or that carry DNA mutations ${ }^{2,3}$. There are three stages of apoptosis in the testis: apoptosis in the fetal period, the first wave of apoptosis during prepuberty and sporadic apoptosis throughout adult life ${ }^{5,6}$. In our study, the Magea-null mice showed smaller testes and significantly increased apoptosis in the first wave of spermatogenesis. The first wave of apoptosis at the postnatal age of 2 weeks plays an essential role in spermatogenesis ${ }^{5}$, and disruption of the balance between survival and death results in spermatogenic dysfunction during adulthood ${ }^{4-6}$. The first wave of apoptosis is required for maintaining a critical ratio between the number of Sertoli cells and the number of maturing germ cells and for the removal of spermatocytes with mutated DNA or incorrect genomic rearrangements ${ }^{2,4-6}$. Strikingly, the elevated protein levels of p53 and Bax at the postnatal age of 1 week until $4^{\text {th }}$ week were suggested to trigger the first wave of apoptosis to eliminate cells with DNA lesions $s^{5}$. Recent studies have shown that various types of cancer cells benefit from the activation of the MAGE-A family and gain a survival advantage by inhibiting the $\mathrm{p} 53$ signalling pathway and suppressing $\mathrm{p} 53$-dependent apoptosis via ubiquitination and degradation of $\mathrm{p} 53$ protein $^{19-24,39,40}$. Our results suggested that the Magea gene 
A

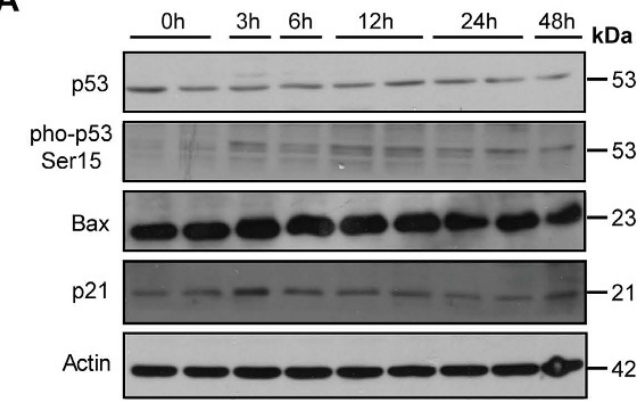

D

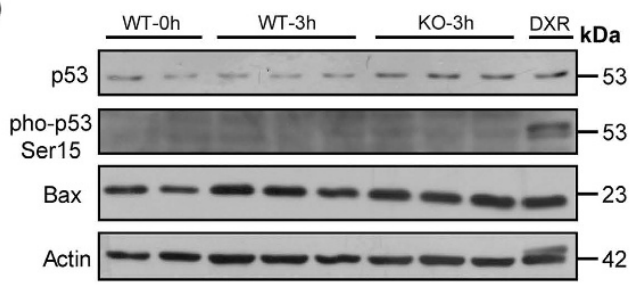

$\mathbf{F}$

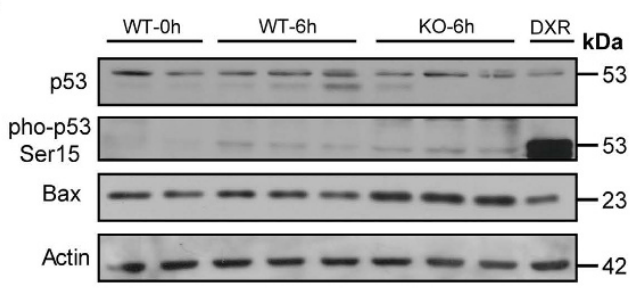

B

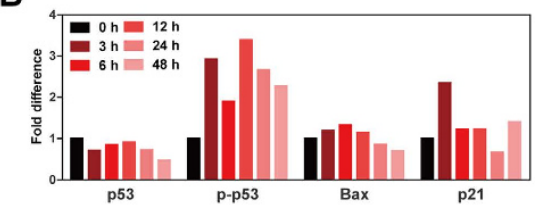

C

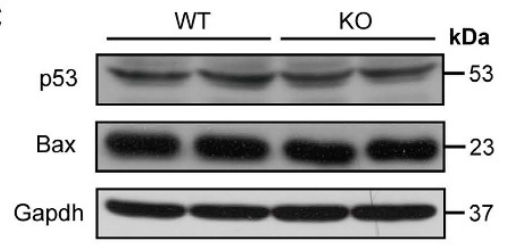

E

ENU 3h

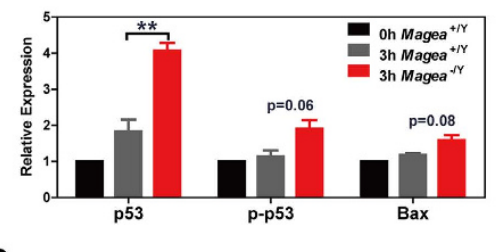

ENU 6h

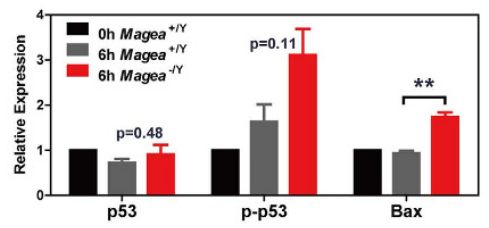

Figure 5. Magea proteins inhibit p53 protein expression after genotoxic stress. (A) Time course analysis of the protein expression of p53 downstream targets in the testes of C57BL/6J mice after exposure to $60 \mathrm{mg} / \mathrm{kg}$ ENU. (B) Quantification of the western blot results. The graphic shows the fold change versus the zero time point after $\beta$-actin normalization. The grey value was calculated using the integrated density value (mean grey value $\times$ area). (C) p53 protein expression in wild-type (WT) and Magea $^{-/ Y}$ (KO) mice. (D) Western blot analysis of the p53 signalling pathway in testes 3 hours after exposure to ENU $(60 \mathrm{mg} / \mathrm{kg})$. WT, wild-type mice; KO, Magea-null mice. DXR, doxorubicin-treated $(1 \mu \mathrm{mol} / \mathrm{L}) \mathrm{NIH} 3 \mathrm{~T} 3$ cells as a positive control. (E) Quantification of the western blot results at the 3-hour time point after ENU treatment. (F) Western blot analysis of the p53 signalling pathway in testes 6 hours after exposure to ENU $(60 \mathrm{mg} / \mathrm{kg})$. (G) Quantification of the western blot results at the 6-hour time point after ENU treatment. The graphic shows the fold change versus the zero time point of wild-type mice after $\beta$-actin normalization. The grey value of the protein band was calculated using the integrated density value (mean grey value $\times$ area). ${ }^{* *} \mathrm{p}<0.01$. The full-length blots are presented in Supplemental Figs S8 and 9.

cluster might have a similar physiological function during normal spermatogenesis. These results raised the possibility that the deletion of Magea genes could activate p53 and/or Bax and thereby induce p53-dependent apoptosis, resulting in an increase in the first wave of spermatogenic apoptosis and a decrease in the size of the testes.

Testicular apoptosis induced by environmental challenges (such as environmental toxicants, temperature influence, dietary changes, or radiation) is essential for eliminating potentially defective or mutant germ cells during spermatogenesis ${ }^{7-10}$. However, excessive apoptosis, resulting from impaired gene regulation, can affect reproductive function and even lead to infertility. Our data showed that after ENU-induced genotoxic stress, silencing of the Magea genes led to excessive apoptosis, increased protein levels of p53 and Bax and increased mRNA levels of apoptosis-related genes, such as Bax, Noxa and Caspase-3. Because p53 mRNA levels in Magea-null testes were comparable to those of the wild-type mice after ENU treatment, we propose that the expression of p53 is not regulated at transcription but rather in a protein degradation manner under genotoxic stress as previously reported. One of the possibilities is that Magea proteins may regulate the stability or degradation of p53 through protein modification ${ }^{20,21}$. The tumour suppressor $\mathrm{p} 53$ is known to play critical roles in germ cell apoptosis under physiological conditions and in response to various stress signals. It serves as one of the guardians of the germ line genome, prevents the offspring from inheriting deleterious mutation by clearing off excessive or defective germ cells. p53 also induces germ cell apoptosis under stresses such as radiation, cryptorchidism, chemotherapeutic drugs and environmental toxicants ${ }^{37,41}$. Our data are consistent with previous studies demonstrating that improper activation of downstream genes or effectors of p53 leads to similar phenotypes of excessive apoptosis in the testes. Overexpression of p53 during spermatogenesis induces excessive apoptosis and impaired differentiation, resulting in testicular defects and consequent infertility ${ }^{41}$. Because p53 is essential in the maintenance of genomic stability during spermatogenesis and should be tightly modulated, there is great interest in the discovery of novel ways for modulating these apoptotic pathways and determining whether the inhibition of p53 signalling could 
promote male germ cell survival under stress conditions. Mdm2 E3 ligase function is essential for controlling the activation extent of p53 in response to DNA damage and the recovery from the p53-mediated stress response $\mathrm{e}^{42}$, and MAGEA2 could inhibit MDM2/MDM4 association and increase MDM4 levels ${ }^{43}$. These findings suggest that MAGEA genes may mediate a coordinated inhibition of p53 function through various biological pathways. In our study, the negative regulation of $\mathrm{p} 53$ and the $\mathrm{p} 53$ signalling pathway by Magea genes highlights the important role of the Magea gene cluster in protecting germ cells from excessive apoptosis under genotoxic stress.

Carlos et al. recently reported that the MAGEA3/6-TRIM28 complex could function as an E3 ubiquitin ligase and degrade AMP-activated protein kinase (AMPK) $)^{19}$. AMPK is a master sensor of energy status that maintains cellular energy homeostasis. Under energy stress, AMPK is activated to suppress anabolic process and promote catabolic metabolism to regain energy homeostasis. Magea genes can also inhibit the function of p53 via protein ubiquitination, conferring chemoresistance to cancer cells and promoting the survival of these cells ${ }^{20,44}$. p53 acts as a key stress sensor that controls cellular growth and death initiation in response to different stress conditions. In the present study, we showed that disruption of Magea genes led to the activation of p53 and increased apoptosis in spermatocytes under genotoxic stress, suggesting that the Magea gene cluster may serve as a stabilizer in protecting germ cells from apoptosis induced by stressors. Furthermore, we showed that the apoptosis in Magea $^{-/ Y}$ testes during the adult age was similar to that of wild-type controls without ENU treatment, suggesting that the role of Magea genes in apoptosis maybe tightly associated with the magnitude of p53 activation. Unlike the high activation state of the p53 signalling pathway during the first wave of spermatogenesis ${ }^{5}$ and under ENU-induced genotoxic stress ${ }^{33,34}$, the expression of p53 in testes declined from 5 weeks of age and remained a low level throughout the adult age ${ }^{5}$. Therefore, it's difficult to observe an increase in apoptosis in Magea $^{-/ Y}$ testes during the adult age without ENU treatment. Our results, together with previous studies, indicate that the Magea genes could inhibit excessive apoptosis through suppressing stress-stimulated hyper-p53 activity, and support the hypothesis that the Magea genes are highly associated with stress conditions and may be stress-related genes. Thus, we propose that the Magea proteins may protect germ cells from energy stress and activate anabolic biosynthesis of compounds such as fatty acids and proteins by degrading AMPK. Our Magea conditional knockout mice provide a good model for studying the function of Magea genes in spermatogenesis during nutritional stress induced by food restriction or deprivation.

In addition to the testes, the MAGEA proteins (MAGEA1, A2, A3, A4, A6, A10 and A12) are also expressed in differentiated human embryonic stem cells, in the developing nervous system and in migrating primordial germ cells ${ }^{27}$, indicating that they might function during embryonic development. Interestingly, mice with a deletion of the Magea gene cluster were viable, fertile and exhibited normal development and normal testis weight before 1 month of age, suggesting that the deletion of Magea genes may not affect the apoptotic wave in the fetal period. Although we observed phenotypic changes only in the testes of Magea-null mice, other abnormalities might be detected under different conditions, including environmental challenges, or over a different timescale than that analyzed in this study. The lack of a markedly altered phenotype could also be explained by gene redundancy. In this study, we found a compensatory increase in Magea 4 mRNA levels in Magea $^{-/ Y}$ mice, and the observed genetic compensation of Magea4 and Magea10 could have partially masked the impact of gene inactivation in Magea-knockout mice (Supplementary Fig. S2C). We noted that Magea10 was highly expressed in reproductive organs (both the testes and ovaries) (Supplementary Fig. S2D), suggesting that it potentially functions during germ cell development. Thus, targeted deletion of Magea10 might increase our understanding of the function of the MAGE-A family in vivo. The whole testicular sperm reserve was significantly reduced in Magea-null testes (Supplementary Fig. S3A). However, no difference in sperm reserve (expressed as per milligram of testis) was observed between Magea-null testes and wild-type controls (Supplementary Fig. S3B). The sperm count in Magea-null epididymis was slightly lower than that of wild-type controls and showed a decreasing trend, although the data did not reach statistical significance (Supplementary Fig. S5C). It's difficult to observe a very significant output in epididymal sperm quantification possibly due to the weak but significant reduction (approximately $16 \%$ decrease) of the testicular weight (Fig. 1C). Similar phenotypes, such as small testes but normal fertility or sperm count, have been reported in mice with targeted deletions of gene members from the C-terminal EH domain-containing (EHD) protein family (EHD4) ${ }^{45}$, the Pumilio gene family (Pum2) ${ }^{46}$, the protein inhibitor of activated STAT (PIASx) family ${ }^{47}$, and the cytochrome $\mathrm{c}$ family ${ }^{48}$. In addition, other mouse models with gene cluster deletions, such as deletions of the Dickkopf (DKK) family ${ }^{49}$, the testicular haploid expressed gene (Theg) family ${ }^{50}$, and the ADP-ribosylation factor (ARF) family ${ }^{51}$, have shown that specific gene members are dispensable for development or fertility. However, mice with deletions of other members of the EHD protein $(\mathrm{EHD} 1)^{52}$ and the Pumilio gene (Pum1) families ${ }^{53}$ exhibited severe defects in spermatogenesis. Therefore, mice with targeted deletions of individual genes or members of a gene family might exhibit only mild phenotypes; thus, it will be necessary to study gene functions by disrupting the entire gene family. Moreover, the shared MHD of the class I MAGE antigens (MAGE-A, MAGE-B and MAGE-C) ${ }^{12}$ and their common expression patterns in germ cells ${ }^{54}$ raise the possibility that $M A G E-B$ and $M A G E-C$ genes might function similarly to the Magea gene cluster described in our study during germ cell development. Further investigation, including the generation of another knockout mouse model with complete deletion of Magea4 and Magea10, will be necessary to define the exact role of Magea in male germ cell development. Indeed, the emergence of the CRISPR/Cas9 system will enable us to delete ten members of the Magea gene family simultaneously ${ }^{55}$.

In summary, our findings provide the first insights into the physiological function of the MAGE gene family in germ cell development. We demonstrate that six members of the Magea gene cluster (Magea1, a2, a3, a5, a6 and a8) play important roles in testicular development, including roles in the maintenance of normal testis size and the protection of germ cells from excessive apoptosis under physiological conditions or ENU-induced genotoxic stress. Our results strongly support the hypothesis that the expression of Magea genes is not dispensable in the testes or simply a by-product event during spermatogenesis, but rather that Magea genes play a critical role in male germ cell development. 


\begin{abstract}
Materials and Methods
Generation of targeting vectors. The Cre-loxp strategy was used to generate the Magea conditional knockout allele. Two targeting vectors were generated for the deletion. For the upstream targeting construct, an $8.8 \mathrm{~kb}$ homologous arm at the $5^{\prime}$ end of Magea 1 was retrieved from 129/Sv BAC (bMQ421A21, Children's Hospital Oakland Research Institute). A loxp-Neo-loxp selection cassette was cloned to the retrieval vector. For the downstream targeting construct, a $7.7 \mathrm{~kb}$ homologous arm at the $3^{\prime}$ end of Magea6 was retrieved from 129/ Sv BAC (bMQ453L15, Children's Hospital Oakland Research Institute). A $2 \mathrm{~kb}$ cassette containing a loxp site and a PGK-Neo gene flanked by frt were cloned to the retrieval vector. A thymidine kinase cassette was used as a negative selection marker.
\end{abstract}

Generation of double targeted ES cells. The upstream targeting vector was electroporated into W4/129S6 ES cells under G418 selection. Neomycin-resistance clones were picked and screened by PCR analysis and confirmed by Southern blot. Out of the 192 neomycin-resistant clones analyzed, three (B9, H2 and H9) had a homologous recombination event. $\mathrm{H} 2$ targeted clones were electroporated with approximately $25 \mu \mathrm{g}$ Cre expression plasmid pBS185 to delete the neomycin-cassette. Approximately 24 colonies were obtained and confirmed as having successfully neomycin deletion. Then the downstream targeting vector was electroporated into two positive ES clones (A3 and F3) with a non-neomycin allele. 288 neomycin resistant ES cell clones were selected and screened for the correct targeting event. Two independent ES clones (2G and 12D) carrying the double targeted allele were injected into blastocysts from C57BL/6J mice. Chimeric mice were mated with the C57BL/6J wild-type mice to verify germ-line transmission. The F1 progeny female Magea ${ }^{\text {flox/+ }}$ were mated with C57BL/6J male mice to generated Magea $a^{\text {flox/Y }}$ in the F2 generation. To obtain the Magea ${ }^{-/ Y}$ hemizygous mice, Magea flox/+ mice were crossed with EIIa-cre transgenic mice. Primers for the identification of positive targeted ES cells and the genotyping of founders and Magea-null mice are listed in Supplementary Table S4 and Table S5, respectively.

Animals and ENU treatment. For the late response assay, $\mathrm{Magea}^{-/ \mathrm{Y}}$ mice and wild-type controls were injected intraperitoneally with $20 \mathrm{mg} / \mathrm{kg} /$ day of ENU (Sigma-Aldrich) for three days (accumulated dose, $60 \mathrm{mg} / \mathrm{kg}$ of ENU). Histological analysis and apoptosis assays were performed 1 week after the first ENU treatment. For the acute response assay, $\mathrm{Magea}^{-/ Y}$ mice and wild-type controls were injected with $60 \mathrm{mg} / \mathrm{kg}$ of ENU by a single i.p. injection at 3 months of age. The mice were euthanised and used for molecular expression detection and apoptosis assays at 3, 6, 12 and 24 hours after treatment. The Animal Care and Use Committee of the Model Animal Research Center at Nanjing University approved all of the animal studies and animal protocols. All the experiments were performed in accordance with the Guide for the Care and Use of Laboratory Animals of the MARC at Nanjing University. Great effort was made to reduce the total number of mice used and to minimize their suffering. All experiments were conducted on male mice (at least 4 mice for each group) at the age of 3 months.

Histological analysis. After they were dissected and weighed, testes from $\mathrm{Magea}^{-/ \mathrm{Y}}$ and wild-type mice were embedded in paraffin, sectioned, and stained with haematoxylin and eosin (H\&E) using routine methods. Briefly, the testes were harvested and fixed overnight in $4 \%$ paraformaldehyde (dissolved in phosphate buffer solution, $\mathrm{pH}$ 7.4) (Sigma-Aldrich) at $4^{\circ} \mathrm{C}$. Subsequently, the paraformaldehyde-fixed tissue was transferred to $70 \%$ ethanol, dehydrated, embedded in paraffin (Sigma-Aldrich), divided into sections (5- $\mu$ m thickness) and stained with H\&E. Sections were photographed using dotSlide virtual microscopy (Olympus). Morphometric evaluation was performed, and the geometric characteristics of the testicular tissues were assessed using dotSlide software (Olympus), yielding the cross-sectional area, circumference (the length of the tubule boundary), lengths of the maximum and minimum axes, and mean diameter (the distance between two boundary points on a line through the centre of gravity) of each tubule cross section. Only data from circular seminiferous tubule cross sections [shape factor (the area relative to the area of a circle with an equal perimeter), $\left(4 \pi \times\right.$ area/perimeter $\left.{ }^{2}\right)$ values of $\left.\geq 0.8\right]$ were used for subsequent analyses according to previously used method to determine seminiferous tubule morphology ${ }^{56}$.

TUNEL assay. The testes were fixed, embedded in paraffin, and sectioned as described above. Apoptosis was evaluated using a TUNEL FITC Apoptosis Detection Kit (Vazyme, Nanjing, China) according to the manufacturer's protocol. In brief, testis sections were incubated with $20 \mu \mathrm{g} / \mathrm{ml}$ proteinase $\mathrm{K}$ for 20 minutes at room temperature and washed in PBS. Sections were then incubated with terminal deoxynucleotidyl transferase and FITC-12-dUTP at $37^{\circ} \mathrm{C}$ for 1 hour. Finally, the sections were washed in PBS three times and counterstained with Hoechst 33342. Confocal images of TUNEL staining were acquired with a confocal fluorescence microscope (Olympus). Negative and positive staining controls were included in each experiment. Images covering the entire testis section were acquired for quantification of apoptotic cells and apoptotic nuclei were counted. TUNEL-positive cells were counted in 9 testis sections ( 3 sections per testis, 3 mice per genotype). All counting and measurement procedures were performed blindly.

Immunofluorescence staining and immunohistochemistry. For immunofluorescence analysis, paraffin sections were first deparaffinized in xylene twice and subsequently rehydrated through graded ethanol. Antigen retrieval was achieved by immersing sections in $0.01 \mathrm{M}$ sodium citrate $(\mathrm{pH} 6.0)$ at $95^{\circ} \mathrm{C}$ for $30 \mathrm{~min}$. After ambient cooling to room temperature, sections were blocked in blocking buffer (5\% bovine serum albumin, $5 \%$ normal goat serum, and $0.1 \%$ Tween 20 in PBS) and incubated with primary antibodies at $4{ }^{\circ} \mathrm{C}$ overnight. The next day, sections were washed in PBS and incubated with secondary antibody at room temperature for 1 hour. Finally, after washing with PBS, sections were mounted in $50 \%$ glycerol and photographed using confocal microscopy (Olympus). Mouse-anti-mouse SCP3 at 1:200 (Abcam, Cambridge, UK), rabbit-anti-mouse Plzf at 1:200 (Santa Cruz Biotechnology, Santa Cruz, CA) and rabbit-anti-mouse cleaved caspase-3 at 1:200 (Cell Signalling Technology) were used for immunofluorescence. The sections were counterstained with Hoechst 33342 to identify 
nuclei. Immunohistochemistry was also performed on paraffin sections using rabbit-anti-mouse Ki-67 at 1:200 (Cell Signalling Technology Inc., Beverly, MA), stained with the HRP-DAB SPlink Detection Kit (ZSGB-Bio, Beijing, China) and photographed using Dotslide (Olympus). All images were processed with Adobe Illustrator CS5. The incidence of testicular apoptosis was determined by quantification of cleaved caspase-3-positive cells. At least 200 round cross-sectioned seminiferous tubules were recorded from two separate sections of the testis from each male. Three to five wild-type or Magea-null testes at each time point were used for immunostaining. All counting and measurement procedures were performed blindly.

Flow cytometry analysis for assessment of apoptosis. Testicular germ cells were isolated to prepare a monocellular suspension according to previously described methods ${ }^{57}$. In brief, the tunica albuginea was removed under a dissecting microscope and the decapsulated testes were incubated in PBS containing $0.5 \mathrm{mg} / \mathrm{ml}$ collagenase (type IV, Sigma) for 15 minutes at $37^{\circ} \mathrm{C}$ in a shaking water bath. After washing twice with PBS, $0.25 \%$ trypsin (without EDTA) in PBS was added and incubated for 5 minutes at $37^{\circ} \mathrm{C}$. The suspension was gently pipetted to disperse the seminiferous tubules and was filtered through a $100-\mu \mathrm{m}$ nylon mesh, washed twice with PBS, and counted in a hemocytometer. For the testicular apoptosis assay, we used the Annexin V-FITC Apoptosis Detection Kit (Vazyme) according to the manufacturer's protocol. The stained cells were analyzed using a FACSCalibur flow cytometer equipped with Cell-Quest software (Becton Dickinson, San Jose, CA).

Western blot analysis. Testis proteins were extracted using 1\% Nonidet P-40, $50 \mathrm{mM}$ Tris- $\mathrm{HCl}(\mathrm{pH} 8.0)$, $150 \mathrm{mM} \mathrm{NaCl}, 0.5 \% \mathrm{Na}$-deoxycholate, $1 \mathrm{mM}$ phenylmethylsulfonyl fluoride, $1 \mathrm{mM} \mathrm{Na}_{3} \mathrm{VO}_{4}, 1 \mathrm{mM} \mathrm{NaF}$, and protease inhibitors (Cell Signalling Technology). Protein lysates $(25 \mu \mathrm{g})$ were separated by SDS-PAGE and transferred onto PVDF membranes (Merck Millipore). After blocking, the membranes were incubated at $4{ }^{\circ} \mathrm{C}$ overnight with the following primary antibodies: anti-p53 (Abcam), anti-phosphorylated-p53 (Ser15) (Cell Signalling Technology), anti-Bax (Santa Cruz Biotechnology), anti-p21 (Santa Cruz Biotechnology) and anti- $\beta$-actin (Cell Signalling Technology). Band intensities on the western blots were quantified by densitometric analysis using ImageJ software (version $1.47 \mathrm{v}$; National Institutes of Health). The grey value of each protein band was calculated using the integrated density value (mean grey value $\times$ area).

Evaluation of testicular sperm reserve and epididymal sperm. Testicular sperm reserve was estimated using the sonication hemocytometric quantification. Briefly, testes from Magea-null mice and wild-type controls were dissected, and the tunica albuginea was carefully removed. Each testis was sonicated for $30 \mathrm{sec}$ using the Vibracell VCX750 Ultrasonic Cell Disrupter (Sonics, Newtown, USA). After recording the sonicate volume, each sonicate was diluted 1:50 v/v using phosphate buffered saline. Remaining sperm nuclei were counted using hemocytometry. The number counted by the hemocytometer was then multiplied by the volume of sonicates and the dilution rate to obtain the whole testicular sperm reserve (expressed as numbers of spermatozoa nuclei per testis). To investigate sperm motility properties, epididymides of wild-type and mutant hemizygous mice were dissected in sterile Modified HTF Medium (OYA Biotechnology) with $10 \%$ fetal bovine serum. Cauda epididymal sperm were allowed to swim out and incubated for $15 \mathrm{~min}$ at $37^{\circ} \mathrm{C}$. Sperm movement was quantified using a computer-assisted semen analysis (CASA) system (Hamilton Thorne Research Beverly, MA, USA) according to the manufacturer's instructions. The experiment of sperm quantification was repeated using a different method. Specifically, the cauda epididymides of Magea-null mice and wild-type controls were carefully dissected. After sonication, the diluted sperm suspension was quantified using Fully Automated Sperm Analyzer (BEION S3, Shanghai Beion Medical Tech).

Reverse transcription PCR and quantitative real-time PCR. Total RNA was prepared from mouse testes using the RNAiso reagent (TaKaRa, Dalian, China) following the manufacturer's protocol. Total RNA $(1 \mu \mathrm{g})$ was reverse transcribed to cDNA using a first-strand cDNA Synthesis Kit (TaKaRa). Real-time PCR was performed using the StepOnePlus ${ }^{\mathrm{TM}}$ real time PCR system (Applied Biosystems) and SYBR Premix Ex Taq (TaKaRa). Quantification was performed according to the manufacturer's instructions, and the expression of target genes was normalized to the housekeeping gene Gapdh or 36B4. The primers for RT-PCR and real-time PCR used in this study are listed in Supplementary Table S6.

Statistical analysis. The experiments were repeated at least three times and the data are presented as the mean \pm SEM. Student's two-tailed $t$-test was used to assess the differences between groups. ${ }^{* *} \mathrm{p}<0.001$; ${ }^{* *} \mathrm{p}<0.01 ;{ }^{*} \mathrm{p}<0.05$.

\section{References}

1. Martincic, D. S., Virant Klun, I., Zorn, B. \& Vrtovec, H. M. Germ cell apoptosis in the human testis. Pflugers Archiv: European journal of physiology 442, R159-160 (2001).

2. Print, C. G. \& Loveland, K. L. Germ cell suicide: new insights into apoptosis during spermatogenesis. BioEssays: news and reviews in molecular, cellular and developmental biology 22, 423-430, doi: 10.1002/(SICI)1521-1878(200005)22:5 < 423::AIDBIES4 > 3.0.CO;2-0 (2000).

3. Shaha, C., Tripathi, R. \& Mishra, D. P. Male germ cell apoptosis: regulation and biology. Philosophical transactions of the Royal Society of London. Series B, Biological sciences 365, 1501-1515, doi: 10.1098/rstb.2009.0124 (2010).

4. Russell, L. D., Chiarini-Garcia, H., Korsmeyer, S. J. \& Knudson, C. M. Bax-dependent spermatogonia apoptosis is required for testicular development and spermatogenesis. Biol Reprod 66, 950-958 (2002).

5. Rodriguez, I., Ody, C., Araki, K., Garcia, I. \& Vassalli, P. An early and massive wave of germinal cell apoptosis is required for the development of functional spermatogenesis. The EMBO journal 16, 2262-2270, doi: 10.1093/emboj/16.9.2262 (1997).

6. Schaller, C. E. et al. Expression of Aire and the early wave of apoptosis in spermatogenesis. J Immunol 180, 1338-1343 (2008). 
7. Richburg, J. H. The relevance of spontaneous- and chemically-induced alterations in testicular germ cell apoptosis to toxicology. Toxicology letters 112-113, 79-86 (2000).

8. Hasegawa, M., Zhang, Y., Niibe, H., Terry, N. H. \& Meistrich, M. L. Resistance of differentiating spermatogonia to radiation-induced apoptosis and loss in p53-deficient mice. Radiation research 149, 263-270 (1998).

9. Cai, L., Hales, B. F. \& Robaire, B. Induction of apoptosis in the germ cells of adult male rats after exposure to cyclophosphamide. Biol Reprod 56, 1490-1497 (1997).

10. Hikim, A. P. et al. Key apoptotic pathways for heat-induced programmed germ cell death in the testis. Endocrinology 144, 3167-3175, doi: 10.1210/en.2003-0175 (2003).

11. Dunkel, L., Hirvonen, V. \& Erkkila, K. Clinical aspects of male germ cell apoptosis during testis development and spermatogenesis. Cell Death Differ 4, 171-179, doi: 10.1038/sj.cdd.4400234 (1997).

12. Chomez, P. et al. An overview of the MAGE gene family with the identification of all human members of the family. Cancer Res $\mathbf{6 1}$, 5544-5551 (2001).

13. Jungbluth, A. A. et al. Expression of MAGE-antigens in normal tissues and cancer. Int J Cancer 85, 460-465, doi: 10.1002/ (SICI) 1097-0215(20000215) 85:4< 460::AID-IJC3 > 3.0.CO;2-N (2000).

14. van der Bruggen, P. et al. A gene encoding an antigen recognized by cytolytic T lymphocytes on a human melanoma. Science 254, $1643-1647$ (1991).

15. Hofmann, O. et al. Genome-wide analysis of cancer/testis gene expression. Proc Natl Acad Sci USA 105, 20422-20427, doi: 10.1073/ pnas.0810777105 (2008).

16. Kim, J. et al. The clinical significance of MAGEA3 expression in pancreatic cancer. Int J Cancer 118, 2269-2275, doi: 10.1002/ ijc.21656 (2006).

17. Vansteenkiste, J. et al. Adjuvant MAGE-A3 immunotherapy in resected non-small-cell lung cancer: phase II randomized study results. Journal of clinical oncology: official journal of the American Society of Clinical Oncology 31, 2396-2403, doi: 10.1200/ JCO.2012.43.7103 (2013).

18. Batchu, R. B. et al. Efficient lysis of epithelial ovarian cancer cells by MAGE-A3-induced cytotoxic T lymphocytes using rAAV-6 capsid mutant vector. Vaccine 32, 938-943, doi: 10.1016/j.vaccine.2013.12.049 (2014).

19. Pineda, C. T. et al. Degradation of AMPK by a cancer-specific ubiquitin ligase. Cell 160, 715-728, doi: 10.1016/j.cell.2015.01.034 (2015).

20. Monte, M. et al. MAGE-A tumor antigens target p53 transactivation function through histone deacetylase recruitment and confer resistance to chemotherapeutic agents. Proc Natl Acad Sci USA 103, 11160-11165, doi: 10.1073/pnas.0510834103 (2006).

21. Doyle, J. M., Gao, J., Wang, J., Yang, M. \& Potts, P. R. MAGE-RING protein complexes comprise a family of E3 ubiquitin ligases. Molecular cell 39, 963-974, doi: 10.1016/j.molcel.2010.08.029 (2010).

22. Peche, L. Y., Scolz, M., Ladelfa, M. F., Monte, M. \& Schneider, C. MageA2 restrains cellular senescence by targeting the function of PMLIV/p53 axis at the PML-NBs. Cell Death Differ 19, 926-936, doi: 10.1038/cdd.2011.173 (2012).

23. Marcar, L., Maclaine, N. J., Hupp, T. R. \& Meek, D. W. Mage-A cancer/testis antigens inhibit $\mathrm{p} 53$ function by blocking its interaction with chromatin. Cancer Res 70, 10362-10370, doi: 10.1158/0008-5472.CAN-10-1341 (2010).

24. Yang, B. et al. Select cancer testes antigens of the MAGE-A, -B, and -C families are expressed in mast cell lines and promote cell viability in vitro and in vivo. J Invest Dermatol 127, 267-275, doi: 10.1038/sj.jid.5700548 (2007).

25. Clotman, F., De Backer, O., De Plaen, E., Boon, T. \& Picard, J. Cell- and stage-specific expression of mage genes during mouse spermatogenesis. Mamm Genome 11, 696-699, doi: 10.1007/s003350010116 (2000).

26. Mueller, J. L. et al. The mouse $\mathrm{X}$ chromosome is enriched for multicopy testis genes showing postmeiotic expression. Nat Genet 40, 794-799, doi: 10.1038/ng.126 (2008).

27. Gjerstorff, M. F. et al. Distinct GAGE and MAGE-A expression during early human development indicate specific roles in lineage differentiation. Hum Reprod 23, 2194-2201, doi: 10.1093/humrep/den262 (2008).

28. Sang, M., Lian, Y., Zhou, X. \& Shan, B. MAGE-A family: attractive targets for cancer immunotherapy. Vaccine 29, 8496-8500, doi: 10.1016/j.vaccine.2011.09.014 (2011).

29. Lakso, M. et al. Efficient in vivo manipulation of mouse genomic sequences at the zygote stage. Proc Natl Acad Sci USA 93, 5860-5865 (1996).

30. Bellve, A. R. et al. Spermatogenic cells of the prepuberal mouse. Isolation and morphological characterization. The Journal of cell biology 74, 68-85 (1977).

31. Brambilla, G. \& Martelli, A. Genotoxic and carcinogenic risk to humans of drug-nitrite interaction products. Mutation research 635, 17-52, doi: 10.1016/j.mrrev.2006.09.003 (2007).

32. Yin, J., Sun, K. \& Chen, B. Time-dependent toxic effects of N-ethyl-N-nitrosourea on the testes of male C57BL/6J mice: a histological and ultrastructural study. International journal of clinical and experimental pathology 8, 1830-1843 (2015).

33. Katayama, K. et al. Ethylnitrosourea induces apoptosis and growth arrest in the trophoblastic cells of rat placenta. Biol Reprod 67, 431-435 (2002)

34. Katayama, K., Ohtsuka, R., Takai, H., Nakayama, H. \& Doi, K. Expression of p53 and its transcriptional target genes mRNAs in the ethylnitrosourea-induced apoptosis and cell cycle arrest in the fetal central nervous system. Histology and histopathology 17, 715-720 (2002)

35. Lu, W. J., Chapo, J., Roig, I. \& Abrams, J. M. Meiotic recombination provokes functional activation of the p53 regulatory network. Science 328, 1278-1281, doi: 10.1126/science.1185640 (2010).

36. Yin, Y., Stahl, B. C., DeWolf, W. C. \& Morgentaler, A. p53-mediated germ cell quality control in spermatogenesis. Dev Biol 204, 165-171, doi: 10.1006/dbio.1998.9074 (1998).

37. Beumer, T. L. et al. The role of the tumor suppressor p53 in spermatogenesis. Cell Death Differ 5, 669-677, doi: 10.1038/ sj.cdd.4400396 (1998).

38. Sue Marty, M. et al. Initial insights regarding the role of $\mathrm{p} 53$ in maintaining sperm DNA integrity following treatment of mice with ethylnitrosourea or cyclophosphamide. Toxicologic pathology 38, 244-257, doi: 10.1177/0192623309357947 (2010).

39. Yang, B. et al. MAGE-A, mMage-b, and MAGE-C proteins form complexes with KAP1 and suppress p53-dependent apoptosis in MAGE-positive cell lines. Cancer Res 67, 9954-9962, doi: 10.1158/0008-5472.CAN-07-1478 (2007).

40. Nardiello, T. et al. MAGE-A inhibits apoptosis in proliferating myeloma cells through repression of Bax and maintenance of survivin. Clin Cancer Res 17, 4309-4319, doi: 10.1158/1078-0432.CCR-10-1820 (2011).

41. Allemand, I., Anglo, A., Jeantet, A. Y., Cerutti, I. \& May, E. Testicular wild-type p53 expression in transgenic mice induces spermiogenesis alterations ranging from differentiation defects to apoptosis. Oncogene 18, 6521-6530, doi: 10.1038/sj.onc.1203052 (1999).

42. Tollini, L. A., Jin, A., Park, J. \& Zhang, Y. Regulation of p53 by Mdm2 E3 ligase function is dispensable in embryogenesis and development, but essential in response to DNA damage. Cancer cell 26, 235-247, doi: 10.1016/j.ccr.2014.06.006 (2014).

43. Marcar, L. et al. MAGE-A Cancer/Testis Antigens Inhibit MDM2 Ubiquitylation Function and Promote Increased Levels of MDM4. PLoS One 10, e0127713, doi: 10.1371/journal.pone.0127713 (2015).

44. Weeraratne, S. D. et al. miR-34a confers chemosensitivity through modulation of MAGE-A and p53 in medulloblastoma. Neurooncology 13, 165-175, doi: 10.1093/neuonc/noq179 (2011).

45. George, M. et al. Ehd4 is required to attain normal prepubertal testis size but dispensable for fertility in male mice. Genesis $\mathbf{4 8}$, 328-342, doi: 10.1002/dvg.20620 (2010). 
46. Xu, E. Y., Chang, R., Salmon, N. A. \& Reijo Pera, R. A. A gene trap mutation of a murine homolog of the Drosophila stem cell factor Pumilio results in smaller testes but does not affect litter size or fertility. Molecular reproduction and development 74, 912-921, doi: 10.1002/mrd.20687 (2007).

47. Santti, H. et al. Disruption of the murine PIASx gene results in reduced testis weight. Journal of molecular endocrinology 34, 645-654, doi: 10.1677/jme.1.01666(2005).

48. Narisawa, S. et al. Testis-specific cytochrome c-null mice produce functional sperm but undergo early testicular atrophy. Molecular and cellular biology 22, 5554-5562 (2002).

49. Kaneko, K. J., Kohn, M. J., Liu, C. \& DePamphilis, M. L. The acrosomal protein Dickkopf-like 1 (DKKL1) is not essential for fertility. Fertility and sterility 93, 1526-1532, doi: 10.1016/j.fertnstert.2009.06.011 (2010).

50. Mannan, A. U. et al. Male mice lacking the Theg (testicular haploid expressed gene) protein undergo normal spermatogenesis and are fertile. Biol Reprod 69, 788-796, doi: 10.1095/biolreprod.103.017400 (2003).

51. Schurmann, A. et al. Reduced sperm count and normal fertility in male mice with targeted disruption of the ADP-ribosylation factor-like 4 (Arl4) gene. Molecular and cellular biology 22, 2761-2768 (2002).

52. Rainey, M. A. et al. The endocytic recycling regulator EHD1 is essential for spermatogenesis and male fertility in mice. BMC developmental biology 10,37, doi: 10.1186/1471-213X-10-37 (2010).

53. Chen, D. et al. Pumilio 1 suppresses multiple activators of $\mathrm{p} 53$ to safeguard spermatogenesis. Current biology: CB 22, 420-425, doi: 10.1016/j.cub.2012.01.039 (2012).

54. Osterlund, C., Tohonen, V., Forslund, K. O. \& Nordqvist, K. Mage-b4, a novel melanoma antigen (MAGE) gene specifically expressed during germ cell differentiation. Cancer Res 60, 1054-1061 (2000).

55. Wang, H. et al. One-step generation of mice carrying mutations in multiple genes by CRISPR/Cas-mediated genome engineering. Cell 153, 910-918, doi: 10.1016/j.cell.2013.04.025 (2013).

56. Benavides-Garcia, R. et al. Granulocyte colony-stimulating factor prevents loss of spermatogenesis after sterilizing busulfan chemotherapy. Fertility and sterility 103, 270-280 e278, doi: 10.1016/j.fertnstert.2014.09.023 (2015).

57. Jeyaraj, D. A., Grossman, G., Weaver, C. \& Petrusz, P. Dynamics of testicular germ cell proliferation in normal mice and transgenic mice overexpressing rat androgen-binding protein: a flow cytometric evaluation. Biol Reprod 66, 877-885 (2002).

\section{Acknowledgements}

We thank Dr. Xingxu Huang and Dr. Geng Liu for their helpful discussions and critical suggestions. The skillful technical assistance of the animal facility staff is also gratefully acknowledged. This work was supported by National Natural Science Foundation of China (grant 31301217) and the Ministry of Science and Technology of China (grants 2009CB91870, 2014BAI02B01 and 2015BAI08B02).

\section{Author Contributions}

S.H., C.L. and X.G. planned the experiments. S.H., L.X., P.S. and Z.L. performed the experiments. S.H., Z.L. and X.G. wrote the paper.

\section{Additional Information}

Supplementary information accompanies this paper at http://www.nature.com/srep

Competing financial interests: The authors declare no competing financial interests.

How to cite this article: Hou, S. et al. The Magea gene cluster regulates male germ cell apoptosis without affecting the fertility in mice. Sci. Rep. 6, 26735; doi: 10.1038/srep26735 (2016).

(c) (i) This work is licensed under a Creative Commons Attribution 4.0 International License. The images or other third party material in this article are included in the article's Creative Commons license, unless indicated otherwise in the credit line; if the material is not included under the Creative Commons license, users will need to obtain permission from the license holder to reproduce the material. To view a copy of this license, visit http://creativecommons.org/licenses/by/4.0/ 